() 1981 IEEE. Personal use of this material is permitted. However, permission to reprint/republish this material for advertising or promotional purposes or for creating new collective works for resale or redistribution to servers or lists, or to reuse any copyrighted component of this work in other works must be obtained from the IEEE.

IEEE Transactions on Nuclear Science, Vol. NS-28, No. 3, June 1981

\title{
NON INTERCEPTING HIGH RESOLUTION BEAM MONITORS
}

F.B. Kroes, A. Maaskant, T. Sluyk, J.B. Spelt

National Institute for Nuclear and High Energy Physics, section Nuclear Physics (NIKHEF-K)

(formerly: Institute for Nuclear Physics Research ( $\mathrm{Ir}_{\mathrm{r}} \mathrm{O}$ ), Amsterdam, The Netherlands)

\section{Toroid Current Monitor}

\section{Introduction}

In literature we find many articles about beam current transformers for accelerators, designed for their special use. The beam current-monitor developed at IKO distincts itself from the others by a breakthrough in the convential design. The main difference in the design of this toroid current monitor is the idea to split up the frequency spectrum of the beam-pulse signal in two discrete parts.

Each frequency region has its own toroid transformer. This possibility leads to an optimum design of the transformer in combination with the cabling and electronics for the special limited frequency region. At last the outputs of both optimized electronic circuits are added together to deliver the ultimate output response that gives an exact representation of the wholc frcquency spectrum produced by the primary beam-pulse. Monitor specifications are:

Dynamic current range of $10 \mathrm{HA}$ to $50 \mathrm{~mA}$, rise time $100 \mathrm{nsec}$, aroop $1 \%$ at $50 \mu \mathrm{sec}$ and accuracy of $1 \%$.

\section{System design}

A schematic diagram of the toroid monitor including the cabting and electronics is shown in fig. 1. The two toroids are placed outside the beam pipe to reduce the vacuum outgassing problems. The beam pipe is interrupted by a coraric ssclator. This vacuum joint is made by alumina helicoflex rings.

Two types of toroid monitors are now in use. One with an inside beam-hole diameter of $5 \mathrm{~cm}$ and the other with an inside diameter of $10 \mathrm{~cm}$.
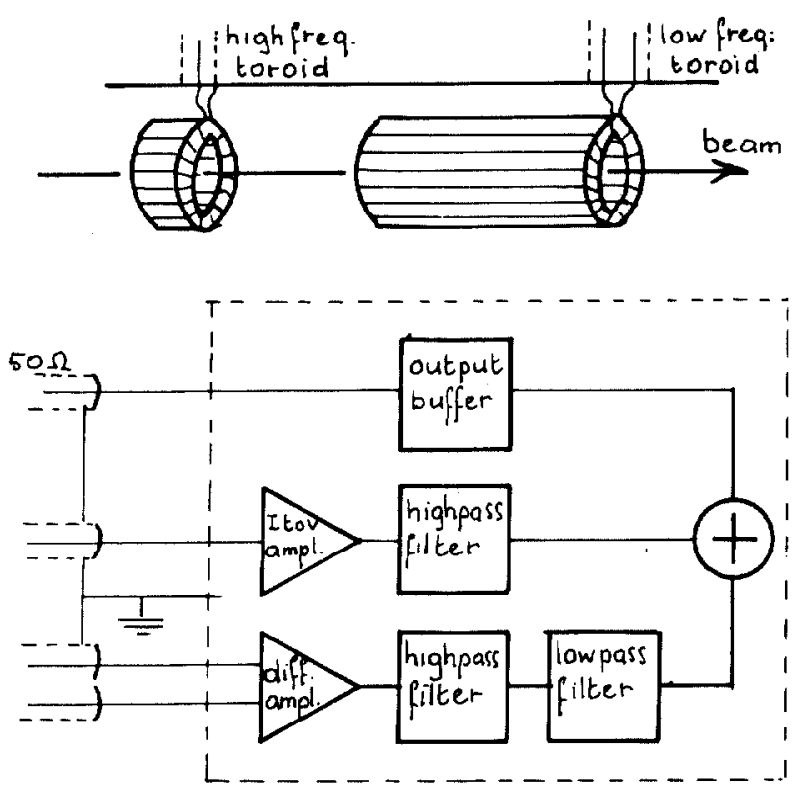

Fig. 1. Toroid current monitor system design
The $10 \mathrm{~cm}$ toroid really exists of:

- A low frequency toroid made of two supermumetal band-wound cores and one $3 \mathrm{~Hz}$ ferroxcube core, stacked together with a 40 turn $0,5 \mathrm{~mm} \mathrm{Cu} / \mathrm{Fm}$ wire winding:

- A high frequency toroid made of one $3 \mathrm{~Hz}$ ferroxcube core with 25 turns of $0,5 \mathrm{~mm} \mathrm{Cu} /$ Em wire.

The $5 \mathrm{~cm}$ toroid exists of:

- A low frequency toroid made ot' tour stacked territe cores with a 100 urn $0,5 \mathrm{~mm} \mathrm{Cu} / \mathrm{Em}$ wire winding.

- A high frequency toroid made of one ferrite core (the same material as used for the low freqrency toroid) with 25 turns of $0,5 \mathrm{~mm} \mathrm{Cu} / \mathrm{Em}$ wire.

The toroids are connected by a triaxial and differential cable of about 25 meters to the electronics in the modulator hall. Care must be taken for shielding and earth problems.

Electronic design

The electronic circuit of the current to voltage amplifiers, filters and adding network is given in fig. 2. Some details of the design vary, devending on the type of toroid used and the number of turns on them. The output of the low frequency toroid is fed by a differential cable, to reduce the $50 \mathrm{~Hz}$ hum, to the differential amplirier. The main reatures of this amplifier are:

- High open loop gain

- Low noise

- High common mode rejection

- High slew rate.

To reduce the low frequency noise, generated by this amplifier, it is followed by a high pass second order active filter with $160 \mathrm{~Hz}$ corner. frequency. A second passive filter limits the frequency band to about $160 \mathrm{kHz}$. The high frequency toroid signal is send through a triaxial cable to the preamplifier and is responsible for the frequency band above $160 \mathrm{KHz}$. So the high frequency amplifier is followed by a high pass active filter with corner frequency of $160 \mathrm{KHz}$.

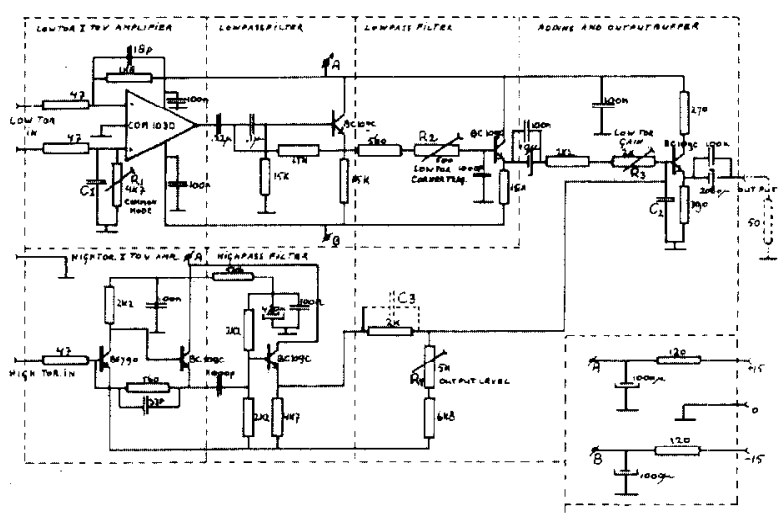

Fig. 2. Electronic circuit Toroid monitor 
A balanced adding of both outputs delivers the actual. output signal over the whole frequency spectrum. After buffering, this signal is ted to a 508 high video data highway for monitoring in the central control room.

\section{Calibration procedure}

First $R_{1}$ is set for meximum common mode rejection. Calibration is achieved by setting the resistors $R_{2}$ to $R_{4}$ and $C_{1}, C_{2}$ according to the next procedure: We feed a current puise through the calibration winding and monitor this pulse on on oscilloscope. The output signal of the toroid current transformer electronics is shown on the same scope. Now $R_{3}$ is set for the rignt balancing between the high-and low frequency amplifier outputs. $R_{2}$ is set for the final high cutoff frequency of the low frequency toroid signal. $R_{4}$ is set for the desired output level. The sensitivity of the monitor must correspond to $10 \mathrm{mV} / \mathrm{mA}$. This resistor at the same time effects both low- and high frequency output signals. $C_{2}$ and $C_{3}$ are set for the final rise time. Because of some spreading in the total combination of the toroids, cables and electronic circuits, it is important to do the calibration procedure in the final confuguration.

\section{Results}

The final toroid transformer design is shown in fig. 3. There are about 10 toroid monitors installed at the accelerator, begm-switch yard and experimental rooms. They are installed on strotegic points. Just behind the injector and at the end of the accelerator, in front of all bending magnets and experimental targets: These monitors are an indispensable measuring device in determine the exact peak beam current at al 1 these points. Because of the accuracy of this monitors it is possible to detect current loss through the beam transport sustem within a few percent. For higher current loss another device will interrupt the electron gun for safety.

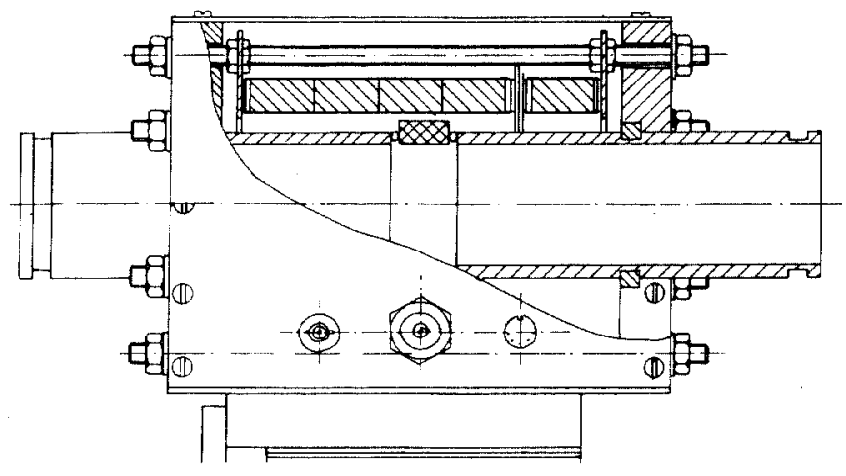

Fig. 3. Final Toroid transformer design

\section{Travelling wave position monitor}

\section{Introduction}

The X-Y- $\not$ SLAC-type cavity position monitor is a very expensive monitor to manufactor. For the lower peak currents of $1 \mu \mathrm{A}$ to $10 \mathrm{~mA}$ used at IKo this monitor needs a R.F. signal processing system to upgrade the monitor sensitivity. Other experiments at SLAC with a phase sensing position monitor of higher sensitivity seemed to be a better solution to IKO's demands. This position monitor transducer is easier to manufactor and finally demonstrates extreme sensitivity and high position resolution in combination with the electronics. Exact center and beam displacement is defined by calibration. Calibration is possible at every moment.

\section{Theory}

The transducer part of this monitor is a simple length of S-band wave-guide with the beam apertures centrally placed in the broad walls. Each end of the wave-guide is terminated by a well matched wave-guide to coax transition, so there are no standing waves in the guide. Fig. 4 shows one arm of this position moni. tor principle.

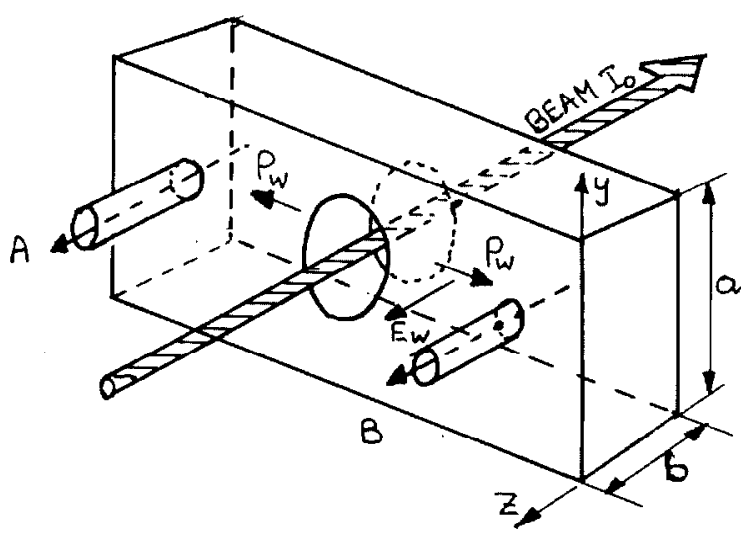

Fig. 4. Travelling wave position monitor principle

A current $I_{0}$ passing tinrough the aperture paraliel to the axis, induces a power $\mathrm{P}_{\mathrm{W}}$ flowing in the $\mathrm{TE}_{10}$ mode towards the transitions where:

$$
\mathrm{P}_{\mathrm{W}}=\mathrm{M}^{2} \frac{\mathrm{b}}{\mathrm{a}} \eta \frac{\lambda_{\mathrm{s}}}{\lambda_{\mathrm{c}}} \mathrm{I}_{0}^{2}
$$

( $n=$ free space irmedance, $\lambda \mathrm{g}=$ guide wave-length, $\lambda_{0}=$ free space wave-length, $M=$ gap transit factor $)$. For S-band wave guide at $2856 \mathrm{MHz} \mathrm{PW}=180 \mu \mathrm{W} /(\mathrm{mA}) \dot{2}$.). Measured at IKO about $100 \mu \mathrm{W} /(\mathrm{mA})^{2}{\overrightarrow{\mathrm{IO}^{2}}}^{2}$ with a beam hole diameter of $4 \mathrm{~cm}$. With the beam centered in a symetrical. structure the signals at $A$ and $B$ are in phase. When the beam moves a distance towards $B$ the phase of the signal at $B$ leads $A$ by $\varnothing$, where

$$
\emptyset=4 \frac{\pi x}{\lambda_{g}} \text { radians. }
$$

at $2856 \mathrm{MHz}$ in S-band wave guide $\frac{d \emptyset}{d x}=4,8$ degrees $/ \mathrm{mm}$. For both horizontal and vertical dx position monitoring, two orthogonal wave-guides connected by a tube of a certain diameter completes the whole monitor transducer. Beam displacement in the y-direction does not alter the phase relation in the $\mathrm{x}$-guide.

\section{System design}

The R.F. signals from the monitor are mixed with a local transistor oscillator signal to produce an intermediate frequency of $10 \mathrm{MHz}$. A trimmer phase-shifter is used in each channel for phase balancing and beam deflection calibration. System setup is shown by fig. 5 . The $10 \mathrm{MHz}$ signals are amplified in limiter-amplifiers, where the amplitude information disappear.

The phase information is cetected by a phase detector and gives the desired beam position information. 


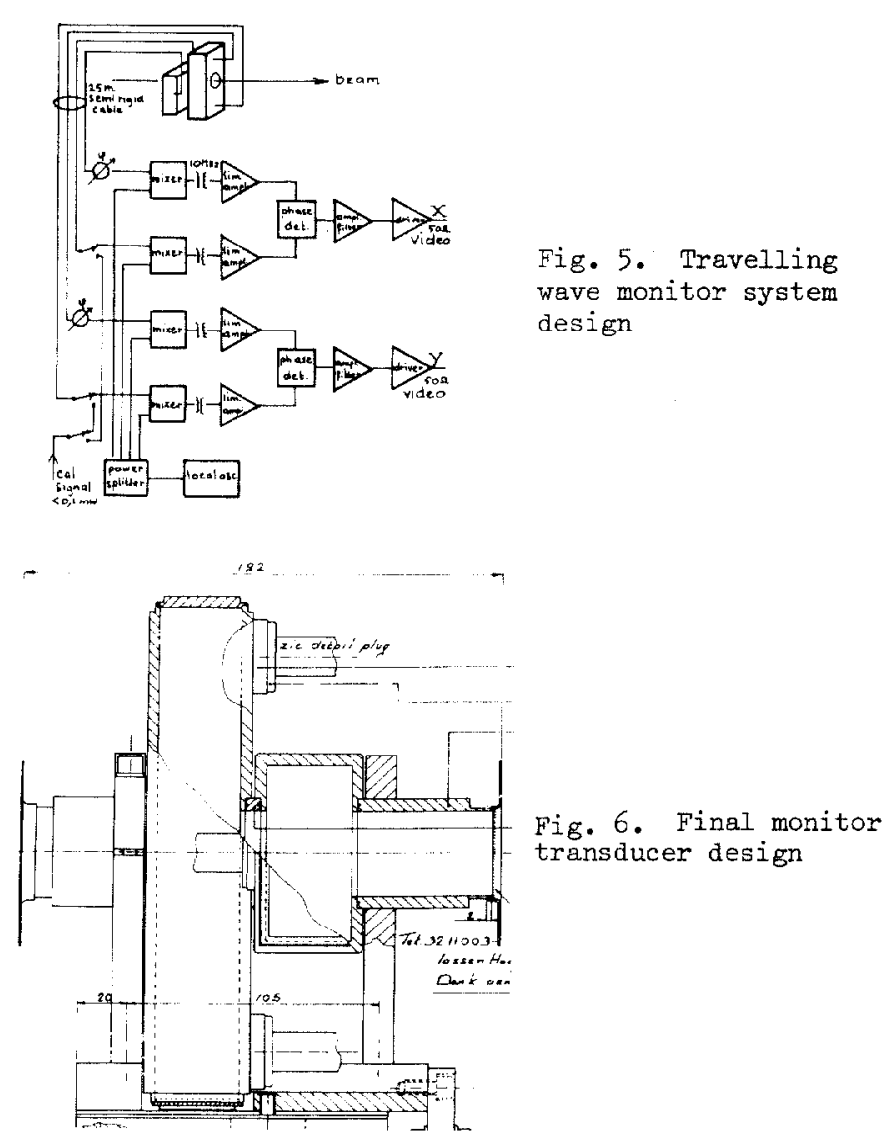

Beam center calibration is possible by feeding a 2856 $\mathrm{MHz}$ test or reference signal into one arm. This signal couples tirrough the beam hole and simulates the beam induced signal in the other guide. With a well made symetrical structure this position corresponds with the exact center of the beam hole. By trimming the phase-shifter in one output it is possible to tune to the correct point of the phase detector response. The same is possible for the other arm. Beam deflection calibraliun is possible if a calibrated phase shifter is used.

\section{Electronic design}

It can be shown that the phase difference between the I.F. outputs of the two mixers is the same as the phase difference between the beam induced microwave inputs. This is true for square lew and linear mixing. The I.F. signals are pessing through two CA 3076 limiter amplifiers with a lower limiting threshold of $50 \mathrm{H}$ Volt. These I.C.'s perform the function of current normalization, since their clipped output wave f'orms carry the phase (position) information independent of input amplitude (current). With an amplifier input impedance of 200 , the minimum I.F. power required to drive the 3076's to the lower limiting threshold is $12,5 \mathrm{p}$ Watt. Using the measured ohtained R.F. power induction rate of $100 \mu \mathrm{W} /(\mathrm{mA})^{2}$ and assuming $\mathrm{aB}$ mixer loss and $3 \mathrm{~dB}$ semi-rigid cable loss, the minimum beam current pulse which will give a usable position signal will be $2 \mu \mathrm{A}$. The 3076 amplifier will accept I.F. signals up to 5 Volt. This indicates an upper beam current of over $30 \mathrm{~mA}$. The 3076 amplifiers are followed by an MC 1596 balanced phase detector and low frequency arplifier-filter. A 500 driver delivers the position video signal to the video data highway. This highway transports the video signal to the control room. Sensitivity of this signal is $30 \mathrm{~m}$ Volt/degree or $140 \mathrm{~m} \mathrm{Volt} / \mathrm{mm}$ beam displacement.

\section{Transducer design}

The wave-guide length of both arms $X$ and $Y$ is chosen in a way that all possible resonances are far enough away from the accelerator frequency. It works out to a length of $20 \mathrm{~cm}$ of $\mathrm{S}$-band wave-guide. The wave guide cosx transitions are tuned according to a special procedure. The R.F. coupling between the guides by the beam-pipe hole is the sum of the R.F. coupling between the two guides for zero wall thickness, and the R.F. attenuation in the beam-pipe hole. The attenuation in the beam-pipe hole for the responsible R.F. mode is below the cutoff frequency of the used hole diameters. The numbers for several hole diameters are:

\begin{tabular}{ccc}
$\begin{array}{c}\text { Hole diam. } \\
\text { in m. }\end{array}$ & $\begin{array}{c}\text { Hole coupling } \\
\text { in } \mathrm{dB}\end{array}$ & $\begin{array}{c}\text { Beam-pipe hole att. } \\
\text { in } \mathrm{dB} / \mathrm{mm}\end{array}$ \\
\cline { 2 - 3 } 20 & -31.96 & 2.02 \\
30 & -21.39 & 1.29 \\
40 & -13.90 & 0.92 \\
50 & -8.09 & 0.65
\end{tabular}

The choice of the beam-pipe length is determined by: - the desired isolation between the two guides

- the used beam-pipe diameter

Chosen is for an isolation of about $25 \mathrm{~dB}$ between the two guides based on:

- the influence of the X-channel to the Y-channel must be low enough

- the calibration signal coupled through the beam-pipe hole must be high enough, starting with a moderate reference signal in the modulator hall.

\section{Results}

The monitor transducer is a totally brazed unit. Materials used are stainless steel and of HC copper. The coax feedthroughs are from alumina oxide. A drawing of the final monitor is given in fig. 6 . The endplates of the guides are tig welded in place after the tuning procedure. Between the transducer in the beam center Iine and the electronics in the modulator hall there are four $\frac{1}{2}$ " prodlines from spiroline about 20 meters long. These semi-rigid cables are bounded together to minimize phase differences caused by temperature variations. Because of thc massive outer conductors it is impossible to radiate microwave power into these cables from neighbouring R.F. cables. Care must be taken to prevent every possibility of radiating R.F. power into this measuring system because of its extreme sensitivity. There is no need to thermostrate the wave-guide transducer part, because it is not a high $Q$ resonating system. Special care must be taken into account of the limiting amplifiers of the channels with rerpect to equalness and noise performance. About 25 position monitors are built of this type now. They are instalied at the accelerator, beam switch yard and experimental rooms, and bave demonstrated their extreme sensitivity and position resolution. This position monitor is demonstrated at M.I.T. Bates Accelerator during 1980 because of their interest, in a very sensitive position monitor with high position resolution. M.I.T. Bates has decided to build the same monitor for their beam recirculator, based on specifications and costs.

\section{References}

1. H. Hogg, D. Farkas

"Some notes on beam-position monitoring devices, and comments on their application to R.L.A."

R.L.A. 38 SLAC

2. Private SLAC communication 